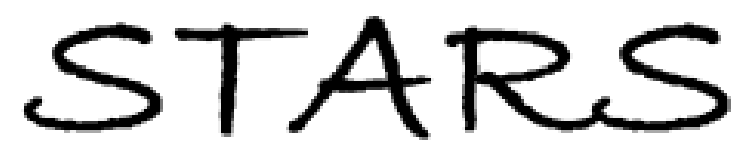

University of Central Florida

STARS

$1-1-2002$

\title{
Reflective liquid-crystal display using an in-plane-switching super- twisted nematic cell
}

Yubao Sun

Hongmei Ma

Zhidong Zhang

Shin-Tson Wu

University of Central Florida

Find similar works at: https://stars.library.ucf.edu/facultybib2000

University of Central Florida Libraries http://library.ucf.edu

This Article is brought to you for free and open access by the Faculty Bibliography at STARS. It has been accepted for inclusion in Faculty Bibliography 2000 s by an authorized administrator of STARS. For more information, please contact STARS@ucf.edu.

\section{Recommended Citation}

Sun, Yubao; Ma, Hongmei; Zhang, Zhidong; and Wu, Shin-Tson, "Reflective liquid-crystal display using an in-plane-switching super-twisted nematic cell" (2002). Faculty Bibliography 2000s. 3495.

https://stars.library.ucf.edu/facultybib2000/3495

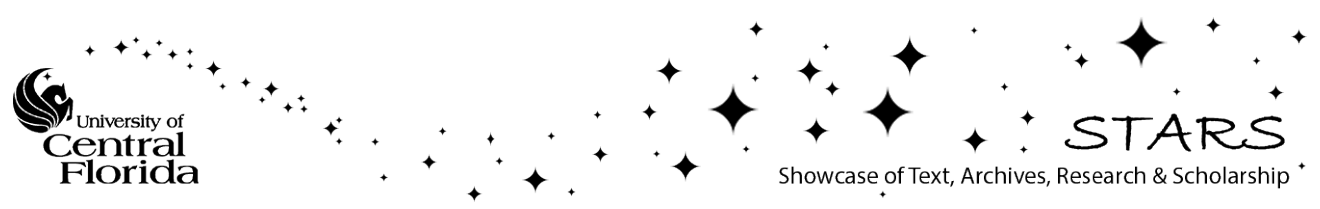




\section{Reflective liquid-crystal display using an in- plane-switching super-twisted nematic cell}

Cite as: Journal of Applied Physics 92, 1956 (2002); https://doi.org/10.1063/1.1496127

Submitted: 13 February 2002 . Accepted: 01 June 2002 . Published Online: 30 July 2002

Yubao Sun, Hongmei Ma, Zhidong Zhang, and Shin-Tson Wu

\section{ARTICLES YOU MAY BE INTERESTED IN}

Response mechanism of nematic liquid crystals using the in-plane switching mode Applied Physics Letters 69, 623 (1996); https://doi.org/10.1063/1.117927

Field effects in nematic liquid crystals obtained with interdigital electrodes Journal of Applied Physics 45, 5466 (1974); https://doi.org/10.1063/1.1663263

Switching of negative and positive dielectro-anisotropic liquid crystals by in-plane electric fields

Journal of Applied Physics 82, 528 (1997); https://doi.org/10.1063/1.365611

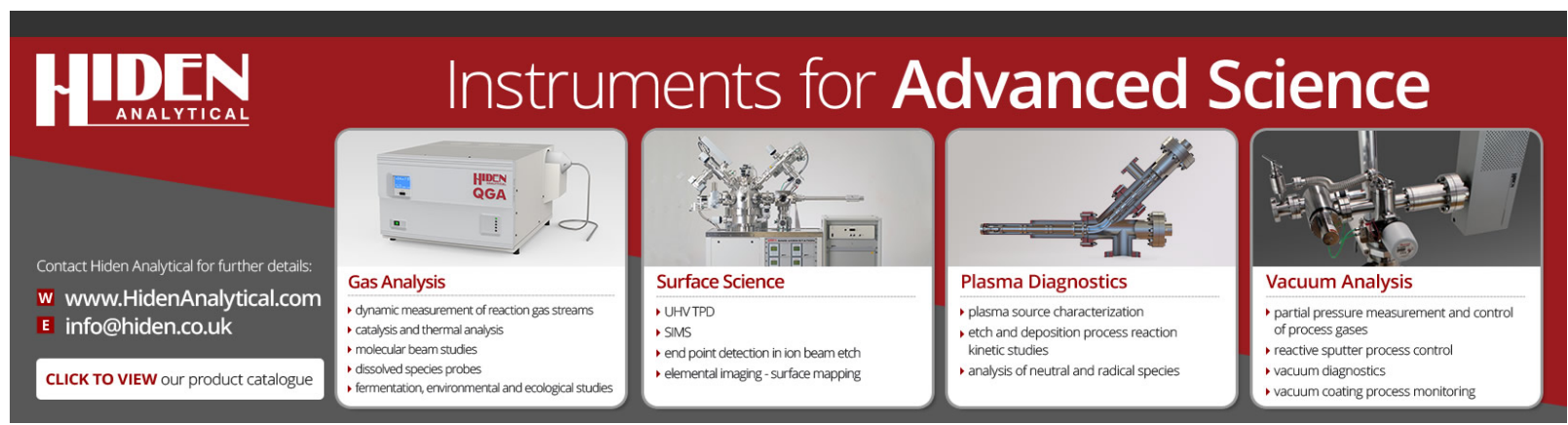




\title{
Reflective liquid-crystal display using an in-plane-switching super-twisted nematic cell
}

\author{
Yubao Sun, Hongmei Ma, and Zhidong Zhang \\ Department of Applied Physics, Hebei University of Technology, Tianjin, 300130, \\ People's Republic of China \\ Shin-Tson $\mathrm{Wu}^{\mathrm{a})}$ \\ School of Optics/CREOL, University of Central Florida, Orlando, Florida 32816
}

(Received 13 February 2002; accepted for publication 1 June 2002)

Two in-plane-switching (IPS) super-twisted nematic (STN) cells are proposed for reflective liquid-crystal displays. The IPS $180^{\circ}$-STN cell exhibits a normally black display with a relatively low-operating voltage, fast response time, and wide viewing angle. Potential applications for large screen reflective displays are foreseeable. (C) 2002 American Institute of Physics.

[DOI: 10.1063/1.1496127]

\section{INTRODUCTION}

Reflective liquid-crystal displays $(\mathrm{LCD})^{1}$ offer several advantages over the transmissive ones in low-power consumption, sunlight readability, and filmlike image quality. First, a reflective LCD uses ambient light for reading the displayed information. Since it does not require a backlight, both power consumption and panel weight are reduced. Second, reflective displays have superb sunlight readability. In a bright outdoor environment, the displayed images of a transmissive LCD could be washed out by sunlight. Because the reflective displays utilize ambient light as a reading source, the brighter the ambient light, the more vivid the displayed images. Third, the thin-film-transistors addressed reflective displays exhibits a large $(90 \%)$ aperture ratio even for highresolution devices. The displayed images have the filmlike quality.

Several reflective LC modes have been developed, e.g., twisted nematic cells with a twist angle ranging from $45^{\circ}$ to $90^{\circ}$ and super-twisted nematic (STN) cells with $180^{\circ}-240^{\circ}$ twist angle. Most of these displays are normally white, i.e., high reflectance at null voltage state. Their viewing angle is around $\pm 40^{\circ}$. Most handheld displays are palm sized so that a $\pm 40^{\circ}$ viewing cone is usually adequate. However, as the panel size keeps on increasing, the demand for wider viewing angle will also increase.

In this article, we utilize the parameter space representation method ${ }^{2,3}$ to search for reflective LC modes that exhibit a wide viewing angle. In Sec. II, a brief description on the theoretical modeling is given. In Sec. III, the simulation results of two reflective in-plane-switching (IPS) modes using the $180^{\circ}$ and $155^{\circ}$ STN cells are presented. The IPS $180^{\circ}$-STN cell exhibits a normally black mode with a wide viewing angle.

\section{THEORETICAL MODELING}

Parameter space is a useful method for designing a LC cell configuration for reflective displays. The geometry of the

${ }^{a)}$ Electronic mail: swu@mail.ucf.edu reflective LCD we studied is shown in Fig. 1. It consists of a polarizer, a quarter-wave film, and a liquid-crystal cell with reflective electrode imbedded in the inner side of the bottom substrate. The axis of the polarizer and the retardation film is at an angle $\alpha$ and $\chi$ with respect to the input director of the liquid-crystal cell. To reduce surface reflections, the polarizer and the phase retardation film can be laminated onto the LC cell. The reflectance $(R)$ of this optical arrangement can be represented by ${ }^{2}$

$$
R=\left|(\cos \alpha, \sin \alpha) F H M(\phi) H^{-1} M(-\phi) F\left(\begin{array}{c}
\cos \alpha \\
\sin \alpha
\end{array}\right)\right|^{2},
$$

where

$$
\begin{aligned}
& H=\left(\begin{array}{cc}
\cos \phi & \sin \phi \\
-\sin \phi & \cos \phi
\end{array}\right), \\
& M=\left(\begin{array}{cc}
A+i B & -C-i D \\
C-i D & A-i B
\end{array}\right),
\end{aligned}
$$

and

$$
F=R_{\chi}^{-1}\left(\begin{array}{cc}
e^{-i \delta} & 0 \\
0 & e^{i \delta}
\end{array}\right) R_{\chi} .
$$

In Eqs. (1)-(4), $F$ is the square of the Jones matrix of the retardation film, and $R_{\chi}$ is the rotation matrix for transforming $F$ to a proper coordinate system, $H M H^{-1}$ represents the Jones matrix of the liquid-crystal cell with light

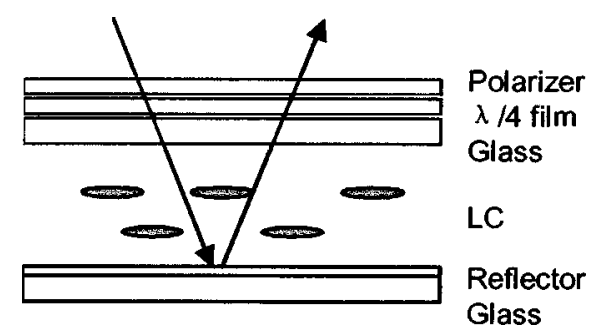

FIG. 1. Device structure of a reflective LCD used for simulations. 


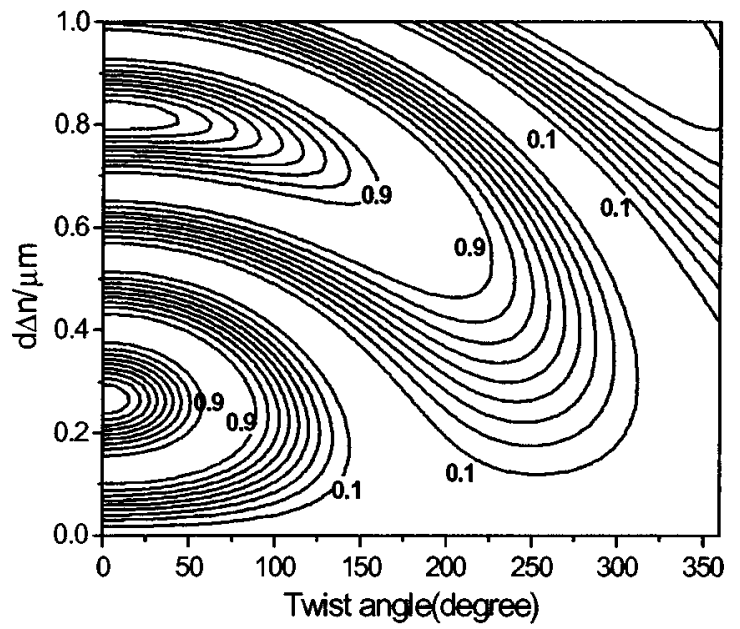

FIG. 2. Contour map of the reflectance of $R-\mathrm{LCD}$ as a function of $d \Delta n$ and twist angle $\phi$.

traveling in the opposite direction and $\phi$ is the twist angle of the liquid-crystal cell. The reflectance of such a display is a unique function of the polarizer's angle $\alpha$, film's angle $\chi$, LC twist angle $\phi$, and the cell gap-birefringence product $d \Delta n$. The reflectance of the LC cell can be plotted as a function of two parameters in the two-dimensional contour map by fixing other two parameters.

Figure 2 shows a contour map of the reflectance as a function of $d \Delta n$ and $\phi$ for the case of $\alpha=0^{0}$ and $\chi=45^{\circ}$. In our calculations, the input light wavelength $\lambda$ is $550 \mathrm{~nm}$ and the LC pretilt angle is $1^{\circ}$. The retardation film is a quarterwave film so that $\delta=\pi / 4$. For the comparison purpose, we only calculate the normalized reflectance of the LC cell; the optical losses from polarizer, quarter-wave film, LC substrates, and reflective electrodes are all neglected. Retaining the angle between the polarizer and the fast axis of the quarter-wave film at $45^{\circ}$, the contour map of the reflectance does not change when we vary the polarizer angle. This is because the incident linearly polarized light is turned to circularly polarized after passing the $\lambda / 4$ film once.

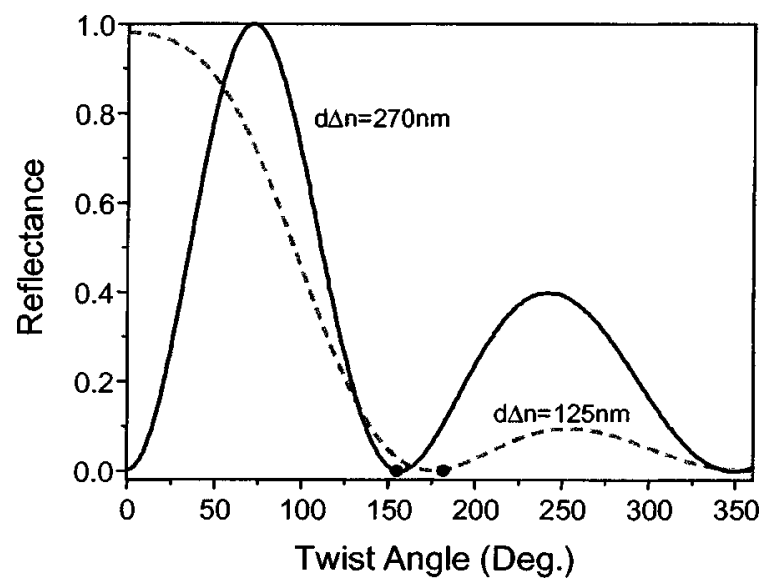

FIG. 3. Twist-angle-dependent reflectance at $d \Delta n=125$ and $270 \mathrm{~nm}$. $R$ $\sim 0$ occurs at $\phi=180^{\circ}$ and $155^{\circ}$, respectively.

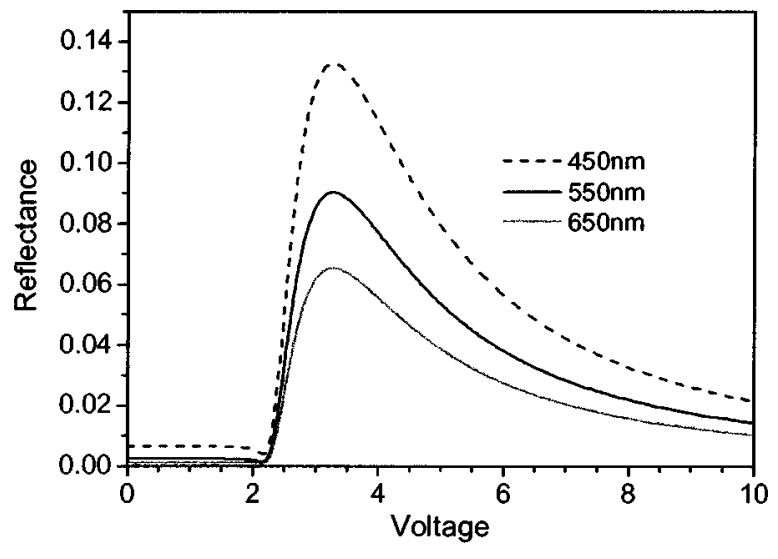

FIG. 4. Voltage-dependent reflectance of the $180^{\circ}-\mathrm{STN}$ cell. $d \Delta n$ $=125 \mathrm{~nm}$. The electric field is in the longitudinal direction.

\section{SIMULATION RESULTS}

The calculated results in Fig. 2 represent the reflectance of a LC cell at the null voltage state. The traces of $R \sim 1$ are potential modes for realizing normally white displays and $R \sim 0$ for normally black. At a given twist angle, the vertical intersections with the contour lines imply to the $d \Delta n$ tolerance of the LC cell. From Fig. 2, two normally white modes $R=1 \quad$ occur at $[\phi, d \Delta n]=\left[63.6^{\circ}, 192 \mathrm{~nm}\right]$, and $\left[191^{\circ}, 583 \mathrm{~nm}\right]$, respectively. These two modes have been discussed and used for reflective light valves. ${ }^{4,5}$ A shortcoming of these two normally white modes is that their contrast ratio is low $(\sim 20: 1)$ because of lacking self-phase compensation. To improve contrast ratio, several mixed-mode twisted nematic (MTN) cells with $60 \leqslant \phi \leqslant 90^{\circ}$ and $\alpha$ $\sim 15^{\circ}-30^{\circ}$ have been developed. ${ }^{1}$

From Fig. 2, we notice that the smallest $d \Delta n$ corresponding to $R \sim 0$ occurs in the vicinities of $[\phi, d \Delta n]$ $=\left[180^{\circ}, 125 \mathrm{~nm}\right]$. Figure 3 plots the twist-angle-dependent reflectance at two $d \Delta n$ values: 125 and $270 \mathrm{~nm}$. From the trace of $d \Delta n=125 \mathrm{~nm}, R \sim 0$ occurs at $\phi \sim 180^{\circ}$. And for $d \Delta n=270 \mathrm{~nm}, R \sim 0$ occurs at $\phi \sim 155^{\circ}$. These are the two normally black modes we report here for the wide view reflective displays.

\section{A. Reflective $180^{\circ}-\mathrm{STN}$ mode}

If we configure the $180^{\circ}-\mathrm{STN}$ cell in a conventional way, i.e., using the longitudinal electric field, the reflectance is less than 15\%, as shown in Fig. 4. The longitudinal electric

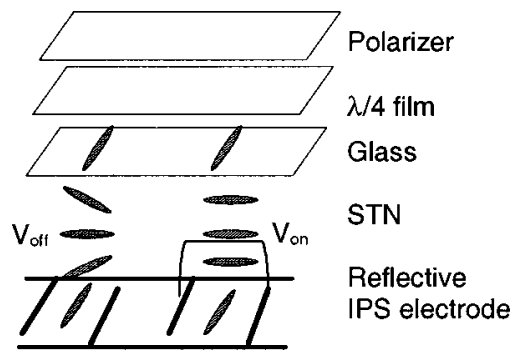

FIG. 5. Device structure of a reflective IPS mode. 


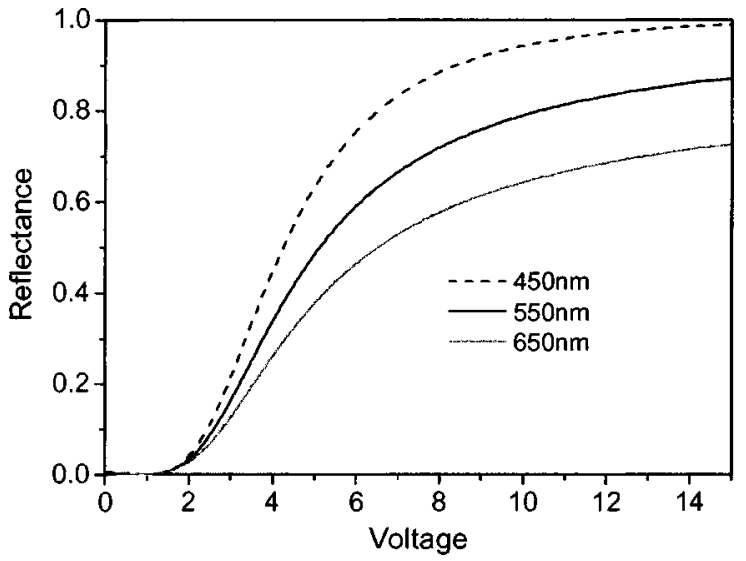

FIG. 6. Voltage-dependent reflectance of the IPS $180^{\circ}-\mathrm{STN}$ cell. $d \Delta n$ $=125 \mathrm{~nm}$.

field is also known to result in a narrow viewing angle. In order to boost reflectance and widen viewing angle, we use the lateral electric field, also called the in-plane-switching (IPS) mode ${ }^{6-10}$ to drive the reflective $180^{\circ}-\mathrm{STN}$ cell.

The reflective IPS cell structure is shown in Fig. 5. In the voltage-off state (left), the LC directors are twisted by $180^{\circ}$. By controlling the $d \Delta n$ of the LC cell properly, a good dark state as shown in Fig. 3 can be obtained. In a voltage-on state, the LC directors are reoriented by the lateral electric field to be parallel to each other, as shown in the right side of Fig. 5. This molecular reorientation retards the phase of the incident and then returned beams. As a result, a bright state occurs.

Figure 6 plots the voltage-dependent reflectance of the reflective IPS $180^{\circ}$-STN cell at three wavelengths: $R=650$, $G=550$, and $B=450 \mathrm{~nm}$. During simulations, the following LC cell parameters are used: $\varepsilon_{\|}=11.4, \varepsilon_{\perp}=3.6, \quad K_{11}$ $=10 \mathrm{pN}, K_{22}=7 \mathrm{pN}, K_{33}=13.6 \mathrm{pN}$, and $10 \mu \mathrm{m}$ electrode gap. Unless otherwise mentioned, the cell gap used for simulations is $d=6 \mu \mathrm{m}$. We vary $\Delta n$ to meet the specified $d \Delta n$ value.

From Fig. 6, a common dark state for RGB colors is observed at $V \leqslant 1.2 \mathrm{~V}_{\mathrm{rms}}$. As the applied voltage increases,

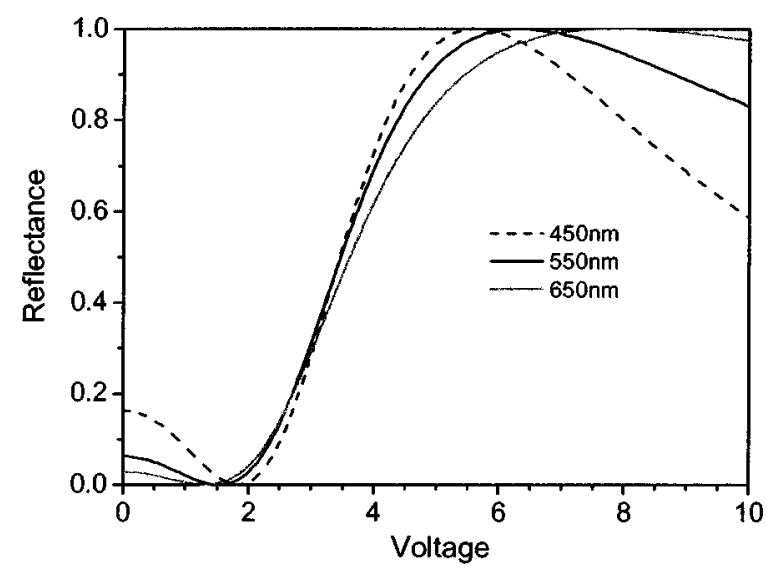

FIG. 7. Voltage-dependent reflectance of the IPS $180^{\circ}-\mathrm{STN}$ cell. $d \Delta n$ $=240 \mathrm{~nm}$.

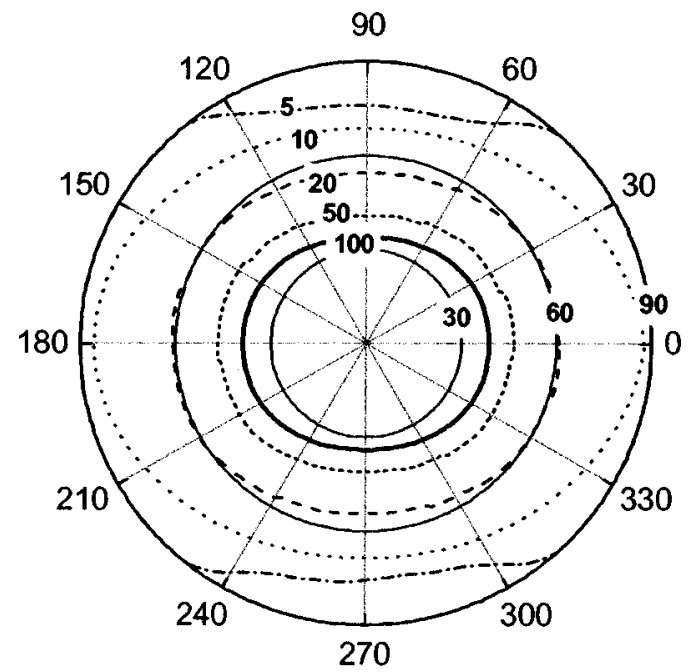

FIG. 8. Iso-contrast contours of the reflective IPS $180^{\circ}$-STN cell. $d \Delta n$ $=240 \mathrm{~nm}$.

reflectance increases. At $15 \mathrm{~V}_{\text {rms }}$, the blue color reaches $R$ $\sim 1$. This operating voltage is too high for most thin-filmtransistor-based liquid-crystal displays.

To reduce the on-state voltage, we could increase either $\Delta \varepsilon$ or $d \Delta n$, reduce the electrode gap, or properly combine these three parameters. Figure 7 repeats the plot as Fig. 6 except for a larger $d \Delta n$ value which is $240 \mathrm{~nm}$. The dark and bright states occur at 1.6 and $6.0 \mathrm{~V}_{\mathrm{rms}}$, respectively. To keep on increasing $d \Delta n$ would lower the bright state voltage further. However, the common dark state for the RGB colors is gradually worsened so that the contrast ratio is reduced. Therefore, an optimal $d \Delta n$ value is around $200-240 \mathrm{~nm}$.

The viewing angle of the reflective IPS $180^{\circ}$-STN cell was calculated. Results are shown in Fig. 8. As expected, the viewing angle exceeds $\pm 80^{\circ}$ at 5:1 contrast ratio. Although the IPS mode offers a very wide viewing angle, there are some tradeoffs, such as reduced aperture ratio, higher onstate voltage, and slower response time. ${ }^{1}$

The present $180^{\circ}$-STN cell only requires $d \Delta n$ $=240 \mathrm{~nm}$, similar to most MTN cells. If the cell gap is maintained at $d=6 \mu \mathrm{m}$, then the required LC $\Delta n=0.04$. The commercially available lowest birefringence TFT-LCD LC mixture has $\Delta n \sim 0.06$. Thus, a proper cell gap is $d=4 \mu \mathrm{m}$

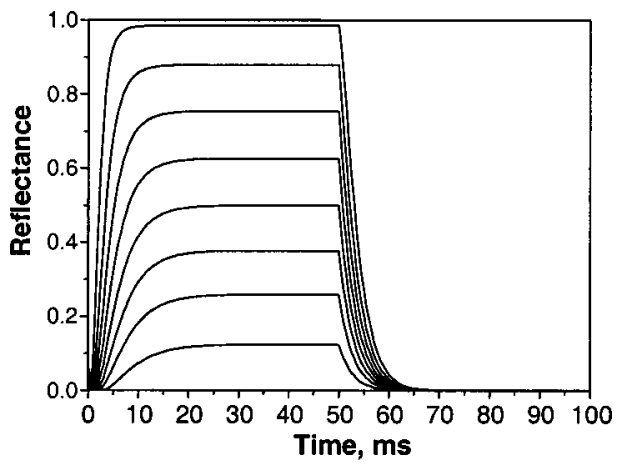

FIG. 9. Calculated response time for the eight-gray levels of the reflective IPS $180^{\circ}-\mathrm{STN}$ cell. $d=4 \mu \mathrm{m}$ and $\Delta n=0.06$. 


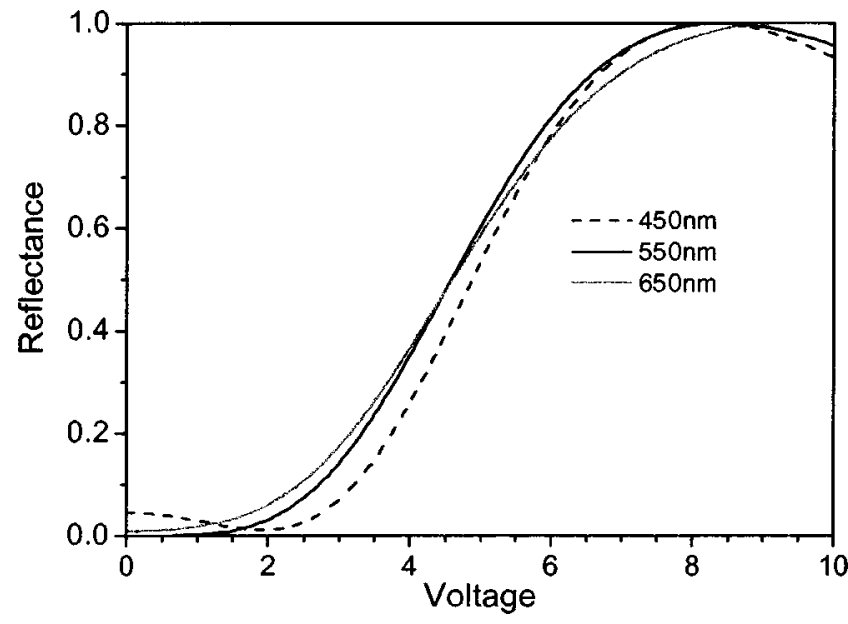

FIG. 10. Voltage-dependent reflectance of the IPS $155^{\circ}$-STN cell. $d \Delta n$ $=270 \mathrm{~nm}$.

which is comparable to a transmissive TN cell. We calculated the response time of the $4-\mu \mathrm{m}$-thick reflective IPS $180^{\circ}$-STN cell at eight gray levels. Results are shown in Fig. 9. For each gray scale, the frame time (rise+decay) is less than 30 ms.

\section{B. Reflective $155^{\circ}-\mathrm{STN}$ mode}

As shown in Fig. 3, another possible normally black mode takes place at $\phi=155^{\circ}$ and $d \Delta n=270 \mathrm{~nm}$. We have also investigated its performance for normally black display.
Figure 10 shows the voltage-dependent reflectance of such a reflective cell in the IPS structure. The dark and bright states occur at 1.5 and $8.0 \mathrm{~V}_{\text {rms }}$, respectively. However, in the dark state, its color dispersion is more severe than that of $180^{\circ}$-STN cell. Thus, the $180^{\circ}$-STN cell would have a higher-contrast ratio.

\section{CONCLUSIONS}

We have demonstrated two in-plane-switching STN cells for reflective liquid-crystal displays. These two reflective displays exhibit a wide viewing angle, relatively low-operating voltage, and fast response time. Potential applications for large screen reflective displays are anticipated.

\section{ACKNOWLEDGMENT}

The UCF group is indebted to AFOSR for financial support under Contract No. F49620-01-1-0377.

${ }^{1}$ S. T. Wu and D. K. Yang, Reflective Liquid Crystal Displays (Wiley-SID, Chichester, 2001).

${ }^{2}$ H. S. Kwok, J. Appl. Phys. 80, 3687 (1996).

${ }^{3}$ S. T. Tang, F. H. Yu, J. Chen, M. Wong, H. C. Huang, and H. S. Kwok, J. Appl. Phys. 81, 5924 (1997).

${ }^{4}$ T. Sonehara and O. Okumura, Proc. SID 31, 145 (1990).

${ }^{5}$ T. Sonehara, Jpn. J. Appl. Phys., Part 2 29, L1231 (1990).

${ }^{6}$ R. A. Soref, Appl. Phys. Lett. 22, 165 (1973).

${ }^{7}$ R. A. Soref, J. Appl. Phys. 45, 5466 (1974).

${ }^{8}$ R. Kiefer, B. Weber, F. Windscheid, and G. Baur, Japan Displays' 92, 547 (1992).

${ }^{9}$ M. Oh-e and K. Kondo, Appl. Phys. Lett. 67, 3895 (1995).

${ }^{10}$ M. Oh-e, M. Yoneya, and K. Kondo, J. Appl. Phys. 82, 528 (1997). 